Journal of Computer Science 4 (7): 557-564, 2008

ISSN 1549-3636

(C) 2008 Science Publications

\title{
Human Computer Interaction Approach in Developing Customer Relationship Management
}

\author{
Mohd Hairul Nizam M. Nasir, Mustaffa Kamal and Wan Ayuni Wan Rozali \\ Faculty of Computer Science and Information Technology, \\ University of Malaya, 50603 Kuala Lumpur, Malaysia
}

\begin{abstract}
Problem statement: Many published studies have found that more than $50 \%$ of Customer Relationship Management (CRM) system implementations have failed due to the failure of system usability and does not fulfilled user expectation. This study presented the issues that contributed to the failures of CRM system and proposed a prototype of CRM system developed using Human Computer Interaction approaches in order to resolve the identified issues. Approach: In order to capture the users' requirements, a single in-depth case study of a multinational company was chosen in this research, in which the background, current conditions and environmental interactions were observed, recorded and analyzed for stages of patterns in relation to internal and external influences. Some techniques of blended data gathering which are interviews, naturalistic observation and studying user documentation were employed and then the prototype of CRM system was developed which incorporated User-Centered Design (UCD) approach, Hierarchical Task Analysis (HTA), metaphor and identification of users' behaviors and characteristics. The implementation of these techniques, were then measured in terms of usability. Results: Based on the usability testing conducted, the results showed that most of the users agreed that the system is comfortable to work with by taking the quality attributes of learnability, memorizeablity, utility, sortability, font, visualization, user metaphor, information easy view and color as measurement parameters. Conclusions/Recommendations: By combining all these techniques, a comfort level for the users that leads to user satisfaction and higher usability degree can be achieved in a proposed CRM system. Thus, it was important that the companies should put usability quality attribute into a consideration before developing or procuring CRM system to ensure the implementation successfulness of the CRM system.
\end{abstract}

Key words: Customer Relationship Management (CRM), User-Centered Design (UCD), Hierarchical Task Analysis (HTA), metaphor

\section{INTRODUCTION}

Customers nowadays have a variety of choices and they are also becoming more knowledgeable and demanding. The power has now been shifted to the customer. With this current scenario, companies have realized that they need to treat their customers with care. Companies now have to figure out different ways to manage their customers effectively so that besides acquiring new customers they will also be able to retain their existing customers. The emergence of competition has changed many aspects of existing businesses and generated companies with new business models, business opportunities and processes. Existing companies are being challenged to rethink the most basic business relationship the one between an organization and its customers. To overcome this challenge, many organizations are considering adopting the concept of Customer Relationship Management (CRM).

Customer Relationship Management is a method that integrates the concepts of Knowledge Management, Data Mining and Data Warehousing in order to support the decision-making process to retain long-term and profitable relationships with customers. CRM can be described as a discipline as well as a set of discrete software programmes and technologies which focus on automating and improving the business processes associated with managing customer relationships in the areas of sales, marketing, customer services and support. The purpose of CRM is to help organizations to understand their customers' current

Corresponding Author: Mohd Hairul Nizam M. Nasir, Faculty of Computer Science and Information Technology, University of Malaya, Lembah Pantai, 50603, Kuala Lumpur, Malaysia Tel: +603-79676435 Fax: +603-21784965 
behavior, preferences and future needs. CRM success can be defined in three dimensions ${ }^{[1]}$ :

- Increased profit

- Improved customer satisfaction

- Enhanced customer loyalty

Different orientations have different approaches toward examining customer data ${ }^{[1]}$. There are three approaches involved: profit-centric orientation, customer-understanding orientation and customer relationship orientation.

CRM goes back to the 1980s when the emergence of database marketing contributed to the setting up of customer service groups who would speak individually to the company's entire customer base. The result, more often than not, was failure, for several reasons including lack of organizational discipline, key staff departures prior to system completion and most importantly a belief that computer systems were the answer to CRM woes. CRM failure was the rule rather than the exception for many early adopters, but lessons were learned along the way, including the reality that solid and uniformly embraced sales and business processes drive the relationship and sales effort. Certainly, technology can be critical to the successful support of good processes, but it cannot be the driver. Customers nowadays are becoming more knowledgeable and demanding. With this current scenario, companies have to figure out different ways to manage their customers effectively so that besides acquiring new customers they will also be able to retain their existing customers. To overcome this challenge, many organizations are considering adopting the concept of CRM.

Currently, many CRM vendors seem to get feedback from their users and customers through surveys, the Internet and other methods. Even though the users are being consulted in these processes, this is not a User-Centered Design (UCD). UCD refers specifically to the process of observing the users in action and applying their input to evolve designs. Besides, creating the user experience for the business application is the key to the success of CRM implementation. According to research, Human Computer Interaction (HCI) is critical to the success of CRM. Another technology that has proven to be important is the appearance of the visual interface. It can account for more than $40 \%$ of the development effort for an application. This study will looks into the issues that currently haunt CRM failures. It proposes the concepts shown in Table 1.
Table 1: The concepts proposed in the system

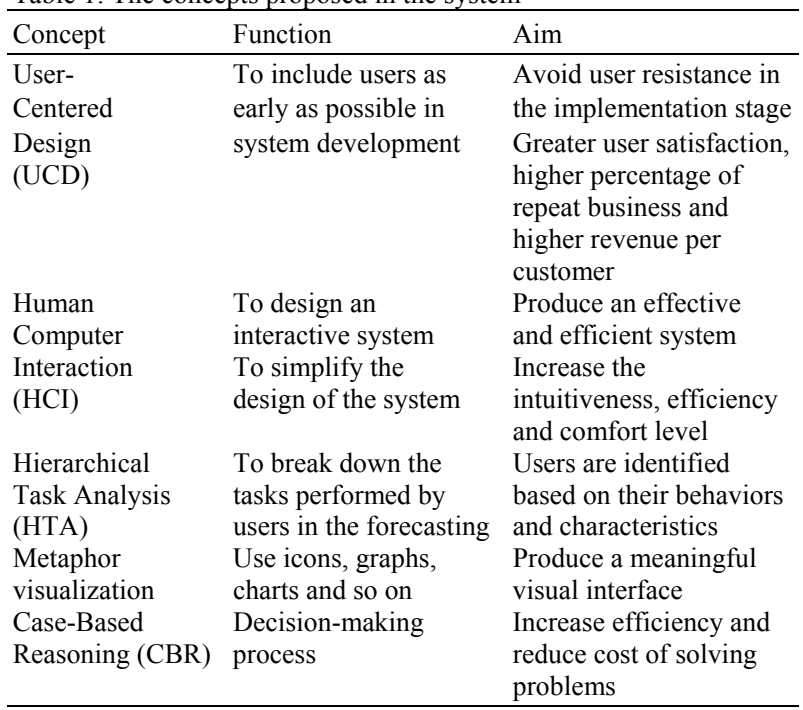

\section{Overview:}

Hierarchical Task Analysis (HTA): HTA is a method that decomposes high-level tasks into subtasks and actions based on graphical structure chart notation. It involves an iterative process of identifying tasks, categorizing them, breaking them down into subtasks and checking their accuracy. It is very useful for HCI work. It entails observations of users: how they work and the steps required to complete tasks.

Human Computer Interaction (HCI): A study conducted by ${ }^{[2]}$ showed that research on HCI has successfully and fundamentally changed the computing world today. The ubiquitous graphical interface of Microsoft Windows 95 was based on Macintosh, which was based on work at Xerox PARC. Even the growth of the World Wide Web is a direct result of HCI research. The now ubiquitous direct manipulation interface where visible objects on the screen are directly manipulated with a pointing device was first demonstrated by Ivan Sutherland on a sketchpad in 1963. Multiple tiled Windows were demonstrated in Engelbart's NLS in 1968. The idea of hypertext is credited to Vannevar Bush's famous MEMEX idea from 1945. Even the User Interface Management System (UIMS) was first created at Imperial College London by William Newman in 1966-7. Therefore we can conclude that all the most important innovations in HCI have benefited from research done by $\mathrm{HCI}$ experts in the early days.

User-Centered Design (UCD): UCD is a comprehensive software development methodology driven by clearly specified, task-oriented business 
objectives and recognition of users' needs, limitations and preferences. There is a need for a user-centered approach in software development that focuses on real users and their goals to drive the development of the product. As a consequence, a well-designed system should make the most of human skill and judgment, should be directly relevant to the work in hand and should support rather than constrain the user. Nowadays, more and more companies are realized the importance of UCD and developing their own UCD processes.

Case-Based Reasoning (CBR): CBR refers to both a cognitive and a computational model of reasoning by analogy with past cases. A basic premise in CBR is that many problems that decision makers encounter are not unique, but rather they are variations of a problem type. It is often more efficient to solve a problem by starting with the solution to a previous, similar problem than to generate the entire solution again from first principles. In fact, experts have been observed to reason through analogy with prior cases. In solving a current problem, CBR (whether it is a human or a computational model) recalls a similar past case and its solution. The reasoning then adapts the successful solution to the recalled case to adjust for any differences between the current case and the recalled case. Finally, the CBR stores the solution to the current case along with feedback about the outcome so that it can be used in solving future problems. Typically, a CBR system consists of a database of past cases and their solutions, a set of indices for retrieving previous cases and storing new cases, a set of rules for measuring similarity and rules for adapting recalled case solutions.

\section{Analysis:}

Issues with CRM systems: A Gartner study found that approximately $55 \%$ of all CRM projects failed to meet software customers' expectations ${ }^{[3]}$. A research found that the failure rate of CRM implementation is estimated to be greater than $65 \% .{ }^{[4]}$ Lowering the failure rate and supporting the success of the system are the ultimate goals of researchers. Below are the identified issues that influence the adoption of CRM and explain why CRM is not adopted fully in both settings.

No ease of use: Most CRM applications are designed with complicated tools and functionality, due to which users face difficulties. Users have to spend more time training as well as mastering the complexity of the processes. Thus the system designed must be exceptionally easy to use and require minimal training.
No understanding from the perspective of the business solution: Software providers normally fail to talk about their technology in the context of a business solution at all. They understand the features and functions, but do not have the capability to see things through the eyes of the customer and communicate how the technology will solve the customer's problem. Thus, when the system is implemented, it leaves the inexperienced client to try out the system and often fails to work with the client's business solution.

Wrong focus: When CRM vendors focus on the benefits of the technology, they focus on the wrong thing. They look for technology that looks powerful but does not pan out in a live implementation.

No user involvement: Lack of user involvement was the second reason for project failure recognized by the Standish CHAOUS Study in 2002. Conversely, it has also been the leading contributor to project success. Even when delivered on time and on budget, a project can fail if it does not meet users' needs or expectations.

User resistance: Based on research, some of the tools in CRM tend to have many features that are too complicated for the users. A study provides a review on CRM implementation showing that one of the failures in CRM implementation is user resistance to adoption. A research conducted by AMR Research on 80 enduser companies found that human resistance to change remains top of CRM buyers' concerns. Some of the users found the system intimidating. These situations show that besides learning about new software, CRM implementation requires a cultural change.

System architecture: Client server architecture was chosen for this project. By using the relational database management system (DBMS), user queries could be answered directly. Furthermore, it also reduced network traffic by providing a query response rather than total file transfer. It improves multi-user updating through a GUI front end to a shared database. In client/server architectures, standard query language (SQL) statements are typically used for communication between the client and server. Two-tier software architecture was chosen for this project. Two-tier architecture is intended to improve usability by supporting a forms-based and user-friendly interface. It also improves scalability by accommodating up to 100 users and improves flexibility by allowing data to be shared. Two-tier architecture requires minimal operator intervention and is frequently used in non-complex, non-time-critical information processing systems. 
As shown in Fig. 1, the two-tier architectures consist of three components distributed in two layers: client (requester of services) and server (provider of services). The three components are:

- User System Interface (such as session, text input, dialog and display management services)

- Processing Management (such as process development, process enactment, process monitoring and process resource services)

- Database Management (such as data and file services)

The two-tier design allocates the user system interface exclusively to the client. It places database management on the server and splits the processing management between client and server, creating two layers. In this project, the entire user interface is placed in the upper tier and the Oracle tables are in the second tier.

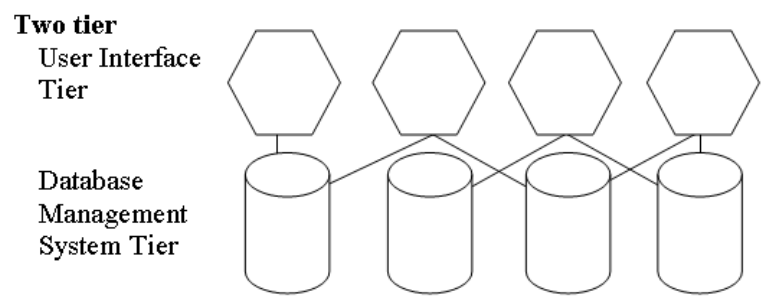

Fig. 1: Two-tier client server architecture design

Table 2: Comparison of CRM software programs

\begin{tabular}{llll}
\hline $\begin{array}{l}\text { Software/ } \\
\text { Features }\end{array}$ & $\begin{array}{l}\text { Salesforce. } \\
\text { com }\end{array}$ & $\begin{array}{l}\text { Siebel CRM } \\
\text { OnDemand }\end{array}$ & $\begin{array}{l}\text { Microsoft } \\
\text { CRM }\end{array}$ \\
\hline $\begin{array}{l}\text { Task tracking } \\
\begin{array}{l}\text { One-click navigation } \\
\text { to any part of application }\end{array}\end{array}$ & 4 & 5 & 4 \\
$\begin{array}{l}\text { Modular deployment } \\
\text { List building on the fly }\end{array}$ & 1 & 3 & 3 \\
for ease of navigation & 4 & 1 & 1 \\
$\begin{array}{l}\text { Simplicity of the user interface } \\
\text { User friendly }\end{array}$ & 3 & 3 & 3 \\
\hline
\end{tabular}

Besides the problems discussed above, the analysis of three CRM software programs from the HCI point of view is shown in Table 2.

A survey was conducted to review the current CRM systems. Three major CRM products were chosen: Salesforce.com, Siebel CRM and Microsoft CRM. A comparison was done based on the HCI perspective, considering factors such as interface, userfriendliness and so on. These features are then rated from 1 to 5. Based on Table 2, it can be concluded that the CRM systems currently on the shelves do not adopt the usability design fully. Their interfaces look very promising and good, but they were not designed to meet the usability goals.

\section{MATERIALS AND METHODS}

The research study was conducted from February 2007 until March 2008 at the Department of Software Engineering, Faculty of Computer Science and Information Technology, University of Malaya. The research project will cover the process of forecasting consolidation carried out by the marketing department from the supplier perspective in order to develop a moderated list for the factory to produce the products demanded by the customers. Essentially, we perform the initial literature review on CRM, looking at a few CRM systems that are available in the market and have been reviewed in order to identify the current features, strengths and weaknesses of the systems from the perspective of Human Computer Interaction (HCI).

Single In-Depth Case Study is chosen as the research method. A case study of a multinational company was chosen in this research, in which the background, current conditions and environmental interactions were observed, recorded and analyzed for stages of patterns in relation to internal and external influences.

Besides that, different types of orientation approach were reviewed to examine the customer data. All the information was collected through the online system, mainly from:

- Online journals

- HCI and CRM books

- CRM resource websites such as business websites, case studies, articles, white papers, proceedings and conference papers

Secondly, some techniques of combined data gathering: interviews, naturalistic observation and studying documentation gathering requirements from the users have been employed. These methods were chosen because there was a need to fully understand the natural activities performed when users were carrying out their tasks, exploring issues related to the process and learning the current standards in the process. In this research project, a case study of a supplier company was also conducted. The company was chosen as a sample as it wanted to adopt the CRM, fitted the criteria as a supplier and also faced the CRM issues discussed above.

\section{RESULTS}

Usability is the extent to which a product can be used by specified users to achieve specified goals with effectiveness, efficiency and satisfaction in a specified context. The usability testing was performed at different stages of the development cycle such as the prototyping stage, design stage and so on. All the feedback gathered 


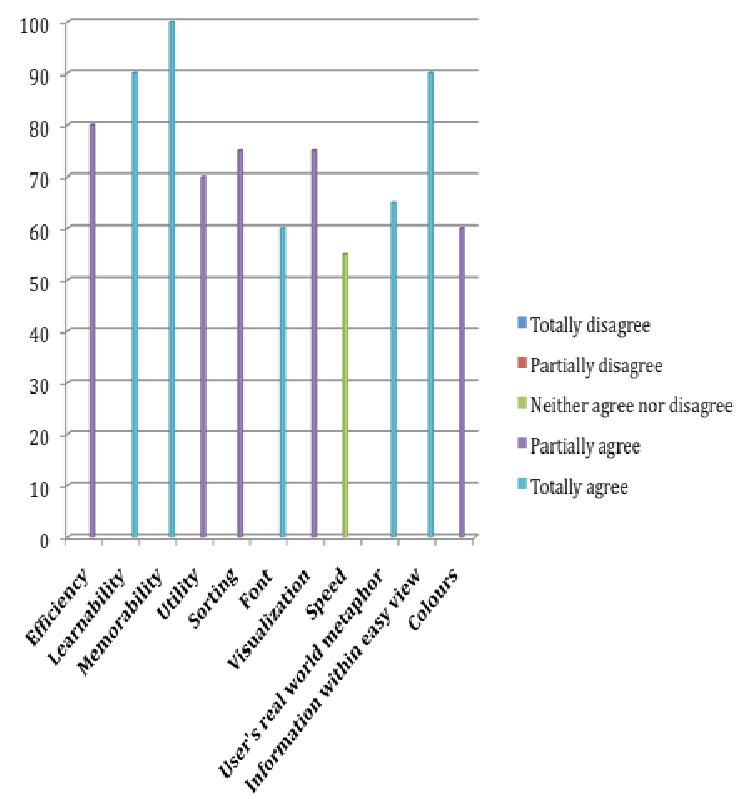

Fig. 2: Results of usability testing

\begin{tabular}{ll} 
Table 3: Acceptance testing status summary & \\
\hline Test & Frequency \\
\hline Executed & 14 \\
Not yet started & 0 \\
Passed first time & 10 \\
Retest required & 4 \\
Retested, OK & 4 \\
Awaiting repair & 0 \\
Completed & 14 \\
\hline
\end{tabular}

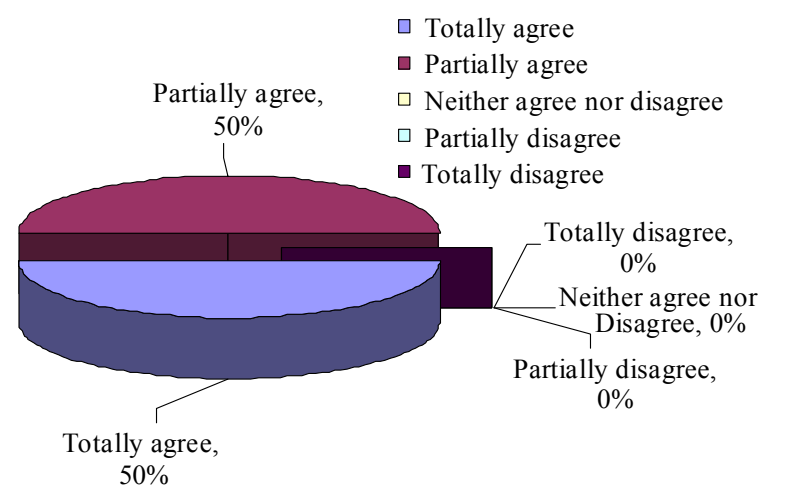

Fig. 3: A sample results from acceptance testing questionnaires

from the session was compiled and incorporated into the system. The final results compiled based on the usability testing questionnaires are shown in Fig. 2.

Acceptance testing is also organized in this system. The purpose of acceptance testing is to obtain feedback from users regarding the system's usability and functionality. It should be able to verify whether the final deliverables meet the users' expectations. The results are shown in Table 3.

According to Fig. 3, the aim of the question is to find out whether users are comfortable using the system after applying the HCI approach to the system. Based on the analysis performed, it is found that all these users agree that the system is comfortable to work with, with $50 \%$ totally agreeing and the other $50 \%$ partially agreeing. From the user acceptance testing, the researcher found that the system developed met the user requirements and expectations and was able to help users to accomplish their tasks efficiently and thus to help the company realize its mission and goal.

\section{DISCUSSION}

The module that is the focus of this proposed CRM system is the sales forecasting module. It provides a tracking mechanism for the sales numbers against stock and probabilities and plans that helps the organization to fine-tune the organization-level sales process. By incorporating the properties mentioned, the proposed HCI approach can lessens the drawbacks of the current CRM system.

Table 4: User involvement in system lifecycle

\begin{tabular}{ll}
\hline System lifecycle & User involvement \\
\hline Requirement gathering & Interview \\
& Naturalistic observation \\
& Studying documentation \\
Analysis & Walk through of use case \\
& User feedback \\
Design & Low fidelity prototyping \\
& High fidelity prototyping \\
& Prototyping evaluation \\
System testing & Developing test plan \\
& System testing \\
& System evaluation \\
System implementation & User survey to get user feedback \\
& System training \\
\hline
\end{tabular}

Simplifying the design of the system: The system incorporates a highly intuitive user interface. This is done by understanding the forecasting process very well, designing alternatives and possibilities and breaking down processes into smaller units. The user would be able to operate the system with only minimal training. The ultimate goal is to make the system as professional looking and easy to use as possible.

Adopting a user-centered approach (UCD): The system is designed to iterate throughout the lifecycle to 
avoid any serious mistakes and to reduce the implementation time. Table 4 shows the degree of user involvement in the proposed system in each phase of the software lifecycle.

By using a user-centered approach (UCD) in which users are involved in every phase of the software development cycle, the issues of no user involvement and user resistance can be addressed.

Using Hierarchical Task Analysis (HTA): HTA was originally designed to identify training needs. In order to develop the HTA for the sales forecast in this project, observations of how users performed their tasks were made. The task decomposition of the forecasting was carried out in the following stages:

- $\quad$ Performing the task of sales forecasting

- Breaking down the forecasting process into subtasks

- Drawing the forecast subtasks in a layered diagram to make sure the subtask is completed

- Deciding which details of the subtasks need to be decomposed. This is to make sure that all the subtask decompositions are treated consistently

- Making sure the decomposition is numbered accordingly

- Presenting the HTA to users who were not involved in the task decomposition but know the tasks well, to check for consistency and accuracy

The use of HTA in the forecasting process will therefore help to address the issue of lack of understanding from the business perspective. Observing how users who actually perform the forecasting process do so in the real world achieves this (Fig 4).

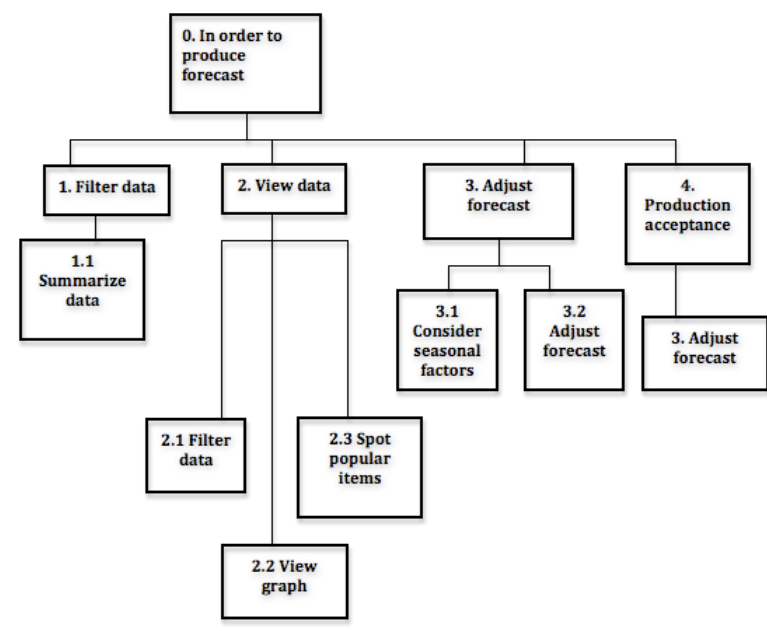

Fig. 4: Graphical representation of HTA of forecasting based on case study

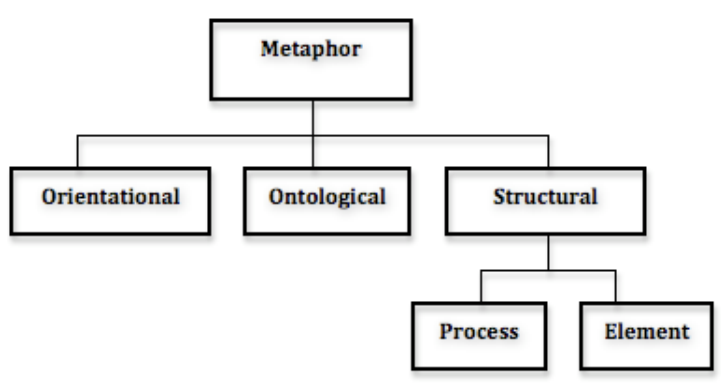

Fig. 5: Taxonomy of user interface metaphor

Use of Metaphor: Metaphor is very popular approach to user interface design. It consists of three components as shown in Fig. 5.

The proposed system is designed for ease of use: icons associated with the operation are the same as the icons used by Microsoft applications. Thus, by looking at the icons, users will know the operations that the system should perform without having to undergo a serious round of training. Thus, it is believed that this will help users to map the unfamiliar knowledge to familiar knowledge. Table 5 described usage of metaphor in the proposed CRM system.

Identify types of users: According to reviews, perceptions of technology and adoption rates vary between types of users. Thus, there is a need to understand types of CRM users and their characteristics and behaviors when performing their tasks. Table 6 shows the user groups identified for the proposed system and their identified tasks. Fig. 6 shows what these user groups wanted from the system.

Table 5: The usage of metaphor in the proposed CRM system

\begin{tabular}{ll}
\hline Metaphors & Usage in the system \\
\hline & The use of "up" and "down" in the date calendar. \\
& "Up" indicates changing the date by going forward \\
& one day and "down," indicates going back one day. \\
& Allows direct manipulation that allows users to \\
& manipulate elements of the user interface, such as the \\
Orientational & mouse. Users are able to scroll using the scroll bar \\
& rather than keyboard-driven commands. But at the \\
& same time, it also has keyboard-only methods of \\
& interaction to allow advanced users to perform tasks \\
& more quickly; for example the short-cut "Control-S" \\
& operates a saving functionality. \\
& Icons used in the main functionality: for example the \\
& "New" functionality is associated with a blank \\
& document and the "Print" functionality is associated \\
& with the printer icon. Besides, all these icons are used \\
& in Microsoft Office and look similar to users who are \\
& also frequent users of Microsoft Office.
\end{tabular}


Table 6: User group table for the proposed system

\begin{tabular}{llc}
\hline User group & Task & No. of users \\
\hline Marketing planner & View forecast & 5 \\
& Consolidate forecast & \\
& Consolidate report & \\
& Moderate forecast & \\
Factory planner & Schedule factory plan & 1 \\
System administrator & Set up master list & 1 \\
\hline
\end{tabular}

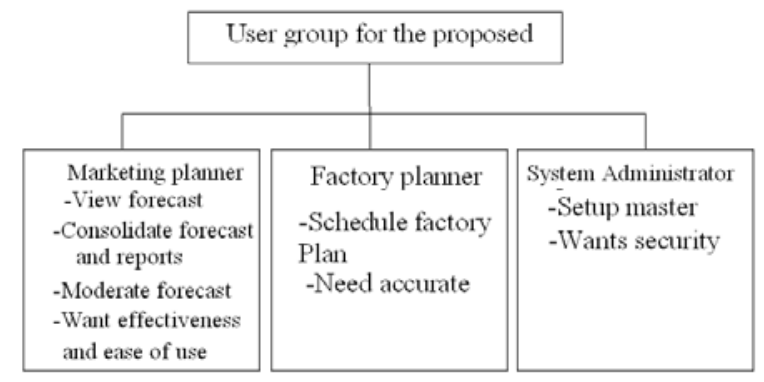

Fig. 6: User groups for the proposed system

Information visualization: This area focuses on the graphical mechanism designed to show the structure of information and improve the cost structure of access. Using data visualization tools can transform the large quantities of complex data into a meaningful visual representation that incorporates science behind the human perception and cognition such as basic charts, graphical indicators, scoreboards, dashboards, advances visualizations, animations and virtual reality environments. This will help the users of information to identify trends and patterns in data that are often not apparent in traditional tabular representations.

CBR design: This is an intelligent system method that enables users to increase efficiency and reduce costs by substantially automating processes. CBR solves new problems by adapting previously successful solutions to similar problems. This project implemented CBR, which consists of major modules: problem input, system engine, indexing rules and lastly output. Problem input is the module where the marketing planner defines the contributors to the decision-making process. It consists of stock, demand, past months' sales and factory capacity. Meanwhile the second module is the system engine, which consists of the following three terms ${ }^{[3]}$ :

- Case base: a database where all the cases are stored

- $\mathrm{CBR}$ inference engine: a computer program that derives the answers from the knowledge base. It is the brain that the expert system uses to reason regarding the information in the knowledge base

- CBR knowledge base: the representation of the cases. The nearest neighbor is implemented in the knowledge base

The next module is the indexing rules and is assigned to each input:

- Indexing rules: consists of assigning the index ID for each contributor code, feature code and case ID, assigning a weight to the contributor code

- Similarity rules: by using the nearest neighbor algorithm for the similarity module, the target case global similarity value will be compared with other global similarity cases in the case base. The matching algorithm is based on the value of global similarity. The equations for local similarity and global similarity are as follows:

$$
\text { Local Similarity }=\sum_{\mathrm{i}=1}^{\mathrm{n}} \mathrm{W}_{\mathrm{i}} \times \operatorname{sim}\left(f_{\mathrm{i}}, f_{\mathrm{i}}^{\mathrm{I}}\right)
$$

Where:

$\mathrm{W}_{\mathrm{i}} \quad=$ The weight of the particular feature

$\operatorname{sim} \quad=$ Similarity function

$f_{\mathrm{i}}^{\mathrm{I}}$ and $f_{\mathrm{i}}^{\mathrm{R}} \quad=$ The values for feature ${ }_{\mathrm{i}}$ in the input and retrieved cases respectively

Global similarity $=$ Total of all local similarities

Later, the global similarity is compared with the other three biggest global similarities that exist in the case base. If the global similarity value is greater than the biggest global similarity value, the new global similarity becomes the new biggest global similarity. If the user inputs that the problem descriptor and the target case global similarity value do not exist in the case base, then he or she needs to update it.

- Adaptation rules: The adaptation rule is to adapt a case to solve the user's problem. The system is capable of using the solution to previous cases to solve the problem presented in the present case where the previous case is similar to the present case. A case consists of two parts: the problem and the solution

- Amendment rules: The marketing planner will review the case in the case base to ensure the solution provided is right. If the solution is not correct and the marketing planner is not satisfied, then he or she will need to amend the solution. The amended version is saved later in the case base, overwriting the old solution 
Finally, the last module is the output whereby the solution/decision of the moderation is shown to the user.

Future enhancement: Based on the study and development experience encountered in this research, it is suggested that the CRM system can be scaled in terms of functionality. A Windows-based application is inadequate for the CRM software. It should be enhanced to make it mobile by building a Web-based application. Web-based applications have many improvements in security and therefore should be able to address the issue of unsecure data. Web-based CRM will be more manageable and easier to deploy, will reduce costs and will provide secure live data. The distribution of products to the customer was not within the scope of this research project. Addressing product distribution will help customers to keep track of their orders and thus will benefit both customers and suppliers. Only static mode is used in viewing graphs. The current features only display tires based on popularity and the zoom facility is not incorporated. It would be more interesting to have live viewing so that users could view details of the tire. Graphical and comprehensive reports should be provided online to benefit users. Availability of comprehensive reports should improve forecast accuracy even if the input sales are limited.

\section{CONCLUSION}

This study highlights the research and development conducted for the CRM system. The current issues have been identified and addressed by implementing the HCI approach. Based on the research conducted, it can be concluded that by using the User-Centered Design (UCD) approach, the Hierarchical Task Analysis (HTA) technique and other Human Computer Interaction (HCI) approaches, it is possible to help users to perform their tasks in the forecasting process efficiently and effectively. Thus, it was possible to develop an interactive and easy-to-use system. Meanwhile, Case-Based Reasoning (CBR) helped the company to automate its decision-making process, which it implemented successfully.

Thus, two main features were proposed which were incorporated in this CRM system. Firstly, in order to increase the usability, the software was developed using the UCD approach and HTA technique in the HCI approach. This stresses the importance of usability in the system and its interfaces are designed to be as simple as possible for ease of use. The system involved users in the early stage of the development work, when their requirements and concerns were gathered and analyzed. By this means it was possible to address the issue of user resistance. Therefore by combining these techniques and approaches a comfort level for the users that leads to user satisfaction is provided.

Secondly, the system incorporates CBR in developing a CRM system for a decision-making process. It solves the problem efficiently by providing a solution to a previous similar problem rather than generating a totally new solution. Thus, it shows the easy-to-use characteristics of the system.

In summary, the system should have easy-to-use characteristics that users will benefit from. Then, it should increase the users' work satisfaction and work productivity thus helping the business to accomplish its mission and goals.

\section{ACKNOWLEDGEMENT}

First and foremost we would like to express our gratitude to the Almighty who made it possible for us to complete the research work successfully. Secondly, we would like to offer our deepest thanks to our colleagues, lecturers and technical staff from the Department of Software Engineering for their endless assistance, technical advice and co-operation.

\section{REFERENCES}

1. Brohman, M.K., R.T. Watson, G. Piccoli and T. Parasuraman, 2003. Data completeness: A key to effective net-based customer service systems. Commun. ACM., 46: 47-51. DOI: $10.1145 / 777313.777339$.

2. Myers, B.A., 1998. A brief history of humancomputer interaction technology. ACM Interactions, 5: 44-54. DOI: $10.1145 / 274430.274436$.

3. Cunningham, C., I.Y. Song and P.P. Chen, 2004. Data warehouse design to support customer relationship management analyses. Proceedings of the 7th ACM International Workshop on Data Warehousing and OLAP, November 12-13, Washington, DC., USA., pp: 14-22.

DOI: $10.1145 / 1031763.1031767$.

4. Kim, H. and S.L. Pan, 2006. Towards a process model of information systems implementation: The case of Customer Relationship Management (CRM). ACM SIGMIS Database, 37: 59-76. DOI: 10.1145/1120501.1120506. 